\title{
Aneurysmal Subarachnoid Hemorrhage Following Intravenous Thrombolysis in Acute Ischemic Stroke
}

\author{
Hyo Jae Kim, MD, Dong-Wha Kang, MD, PhD, Sun U. Kwon, MD, PhD, Jong S. Kim, MD, PhD, Sang-Beom Jeon, MD, PhD \\ Department of Neurology, Asan Medical Center, University of Ulsan College of Medicine, Seoul, Korea
}

\begin{abstract}
Background: An incidental finding of unruptured aneurysm, which is a contraindication to the recombinant tissue plasminogen activator (rtPA), is common in patients with acute ischemic strokes. However, reports describing the rupture of intracranial aneurysm following the administration of rtPA are extremely rare.
\end{abstract}

Case Report: A 51-year-old man presented to the emergency room with global aphasia. A computed tomography (CT) of the brain revealed no intracranial hemorrhage. Since global aphasia occurred in an hour, rtPA was administrated intravenously. A CT angiography was performed 2 hours after an infusion of rtPA, which despite the absence of neurological deterioration and blood pressure surge, revealed subarachnoid hemorrhage in the right cerebral hemisphere, in addition to a 3-mm saccular aneurysm with a bleb in the right middle cerebral artery.

Conclusions: Aneurysmal subarachnoid hemorrhage can develop following the infusion of rtPA. Hence, unruptured aneurysm may not simply be an "incidental finding" in stroke patients receiving $r \mathrm{PA}$

J Neurocrit Care 2017;10(2):107-111

Key words: Cerebral infarction; Thrombolytic therapy; Cerebral aneurysm

\author{
Received July 13, 2017 \\ Revised September 6, 2017 \\ Accepted September 7, 2017 \\ Corresponding Author: \\ Sang-Beom Jeon, MD, PhD \\ Department of Neurology, Asan \\ Medical Center, University of Ulsan \\ College of Medicine, 88 Olympic-ro \\ 43-gil, Songpa-gu, Seoul 05505, Korea \\ Tel: $+82-2-3010-3440$ \\ Fax: +82-2-474-4691 \\ E-mail: sbjeonmd@gmail.com
}

Copyright $\odot 2017$ The Korean Neurocritical Care Society

\section{INTRODUCTION}

The prevalence of intracranial aneurysm is $2 \%$ to $3 \%$ in the general population, and is more common in patients who experience acute ischemic stroke. ${ }^{1-3}$ Intracranial aneurysms have been considered to be a relative contraindication to the administration of the recombinant tissue plasminogen activator (rtPA) due to the theoretical risk for an aneurysmal rupture in individuals who experience acute ischemic stroke. ${ }^{4}$ However, recent studies have not reported an increase in the risk for rtPA-related intracranial hemorrhage in patients with both acute ischemic strokes and intracranial aneurysms..$^{5-7}$ In one prospective multicenter study, the authors provided reassurance that the risk for intracranial hemorrhage does not increase in acute ischemic stroke patients harboring an intracranial aneurysm. ${ }^{5}$ The issue of administering rtPA to stroke patients with an intracranial aneurysm is very important because the evaluation of cerebral vessels to locate aneurysms may result in an in-hospital delay and exclude potential candidates for thrombolysis. Although international guidelines state that an intracranial aneurysm is a contraindication to rtPA, controversy remains as to whether an unruptured aneurysm should be regarded as a contraindication to rtPA,

(cc) This is an Open Access article distributed under the terms of the Creative Commons Attribution Non-Commercial License (http://creativecommons.org/licenses/by$\mathrm{nc} / 4.0$ ) which permits unrestricted non-commercial use, distribution, and reproduction in any medium, provided the original work is properly cited. 
in patients who experience acute ischemic stroke.

To date, case reports describing the rupture of an intracranial aneurysm following the intravenous administration of rtPA are extremely rare and these studies have not convincingly demonstrated that rtPA causes a rupture of the aneurysm. ${ }^{8-10}$ Hence, in routine clinical practice, rtPA tends to be emergently infused in patients exhibiting acute stroke symptoms when a non-contrast computed tomography (CT) detects no intracranial bleeding or any other remarkable contraindications to rtPA.

In this article, we describe a patient who developed aneurysmal subarachnoid hemorrhage (SAH) following the intravenous administration of rtPA for an acute ischemic stroke.

\section{CASE REPORT}

A 51-year-old man with untreated hypertension presented to the emergency room with speech disturbance, which had developed 30 minutes earlier. A neurological examination revealed global aphasia and right hemiparesis. His initial score on the National Institutes of Health Stroke Scale was 9. The standard protocol for acute ischemic stroke in the author's hospital involves a non-contrast CT scan to exclude contraindications to rtPA. Then, after the administration of rtPA, a magnetic resonance imaging (MRI) and a magnetic resonance angiography are performed. ${ }^{11}$

A CT scan of the patient's brain did not reveal an intracranial hemorrhage (Fig. 1A). His initial blood pressure was $205 / 131 \mathrm{mmHg}$, but decreased to $162 / 75 \mathrm{mmHg}$, after the administration of intravenous labetalol. rtPA (0.9 mg/kg) was administered intravenously, 121 minutes after the onset of stroke symptoms. Diffusion-weighted imaging of the brain, which was performed during the infusion of rtPA, revealed acute infarction in the left middle cerebral artery (MCA) territory (Fig. 1B). However, other MRI sequences, including diffusion-weighted imaging, fluid attenuation inversion recovery, $\mathrm{T} 2{ }^{*}$-gradient echo imaging, perfusionweighted $\mathrm{MRI}$, and magnetic resonance angiography, in accordance with the stroke center's protocol, could not be obtained due to poor cooperation from the patient.

A CT angiography was performed, instead of a magnetic angiography, 2 hours after an infusion of rtPA. During this time, the patient did not exhibit any neurological deterioration. Additionally, the patient's blood pressure was controlled to $<180 / 105 \mathrm{mmHg}$, until the CT scan was performed. Blood pressure was measured twice before the CT angiography, with values of $170 / 94 \mathrm{mmHg}$ and 157/77
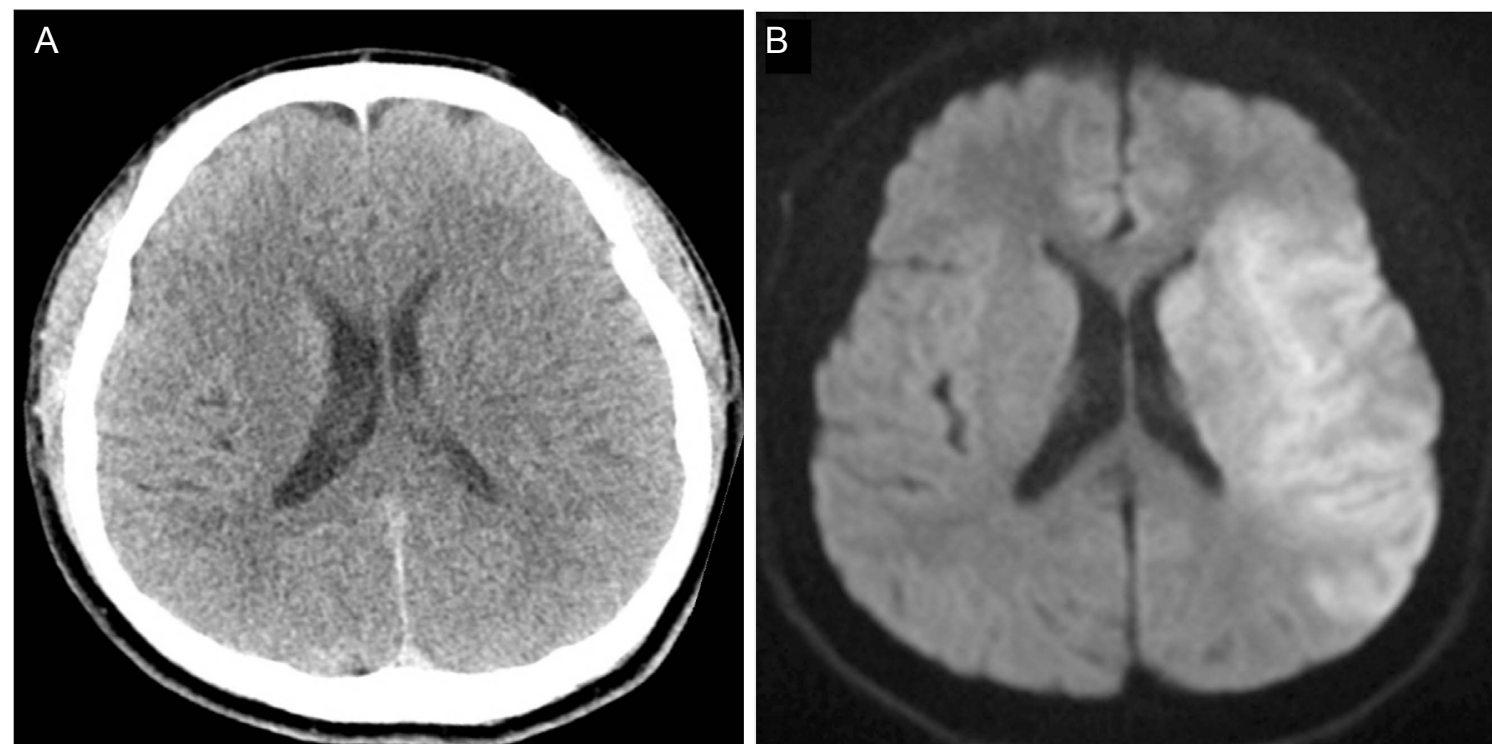

Figure 1. A brain computed tomography, performed before infusion of recombinant tissue plasminogen activator, shows no intracranial hemorrhage (A). Diffusion-weighted imaging shows acute infarction in the left middle cerebral artery territory (B). 
$\mathrm{mmHg}$, and was $169 / 98 \mathrm{mmHg}$ immediately following the $\mathrm{CT}$ angiography. A non-contrast $\mathrm{CT}$, which was performed concurrently with $\mathrm{CT}$ angiography, revealed $\mathrm{SAH}$ predominantly in the right cerebral convexity and falx (Fig. 2A, B). A CT angiography revealed a saccular aneurysm, $3 \mathrm{~mm}$ in size, with a bleb in the right MCA bifurcation and an occlusion of the left MCA M1 portion (Fig. 2C, D). Five hours later, emergent surgical clipping was performed to secure the ruptured aneurysm.
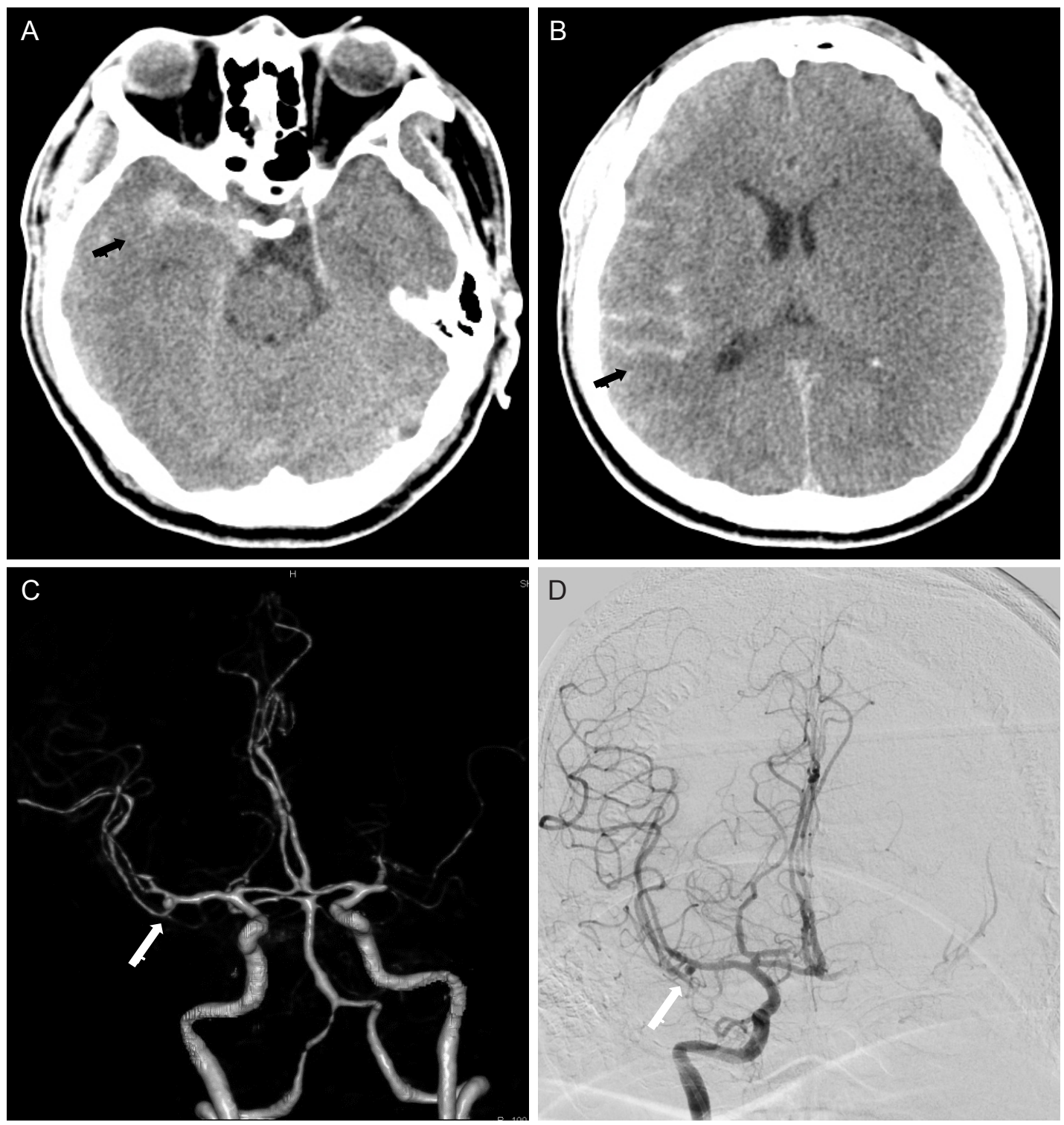

Figure 2. A brain computed tomography (CT) shows subarachnoid hemorrhage predominant in the right cerebral hemisphere (A, B). $\mathrm{CT}$ angiography (C) and conventional angiography (D) reveal a saccular aneurysm with a bleb in the right middle cerebral artery and occlusion of the left middle cerebral artery (black arrows indicate subarachnoid hemorrhage; white arrows, a ruptured aneurysm). 


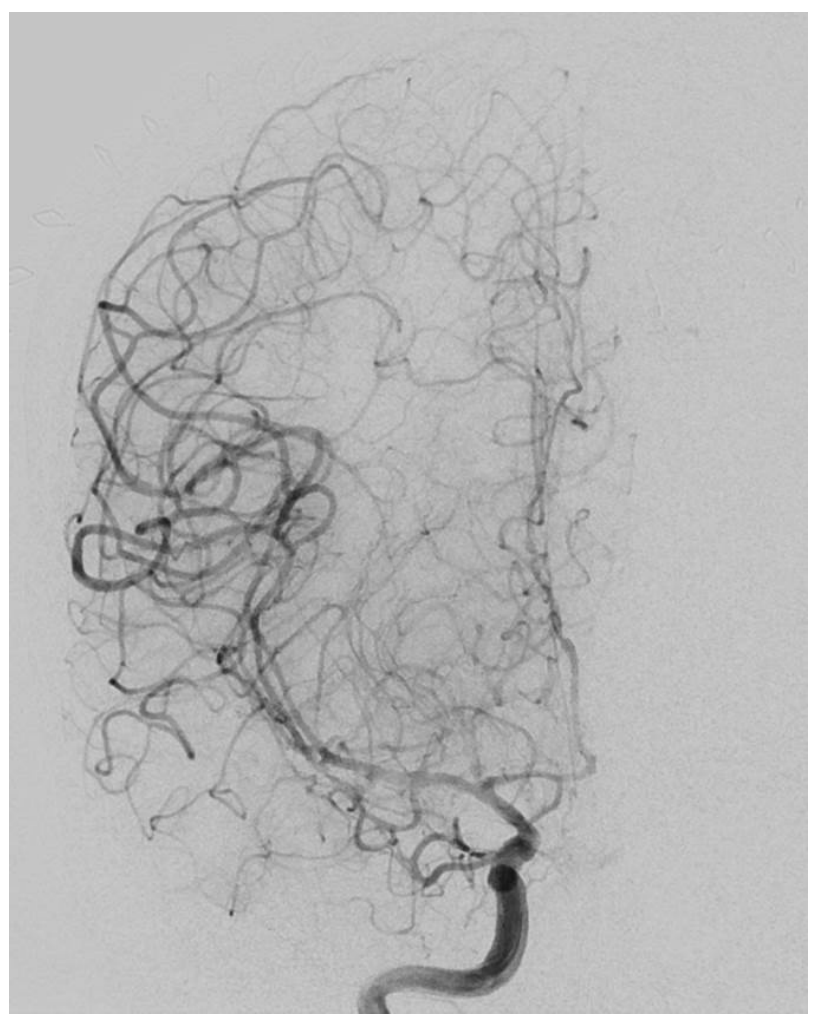

Figure 3. Conventional angiography 7 days after aneurysmal clipping reveals no right middle cerebral artery occlusion or vasospasm.

rysmal clipping. Targeted temperature management $\left(33^{\circ} \mathrm{C}\right)$ was applied for four days due to intracranial hypertension and brain swelling despite hemicraniectomy.

Despite the administration of nimodipine to prevent delayed cerebral infarction, a right MCA territory infarction was detected on a follow-up CT, five days after SAH. However, there were no definitive vasospasms on the conventional angiography (Fig. 3). On discharge five weeks after initial presentation, the patient was alert but had global aphasia and quadriplegia (modified Rankin score 5). His neurological status remained unchanged one year after being discharged.

\section{DISCUSSION}

The patient developed SAH in the right cerebral hemisphere-the contralateral side of the infarction-after ad- ministration of rtPA. A CT angiography revealed a saccular aneurysm with a bleb in the right MCA bifurcation. Thus, we believe that the unruptured aneurysm ruptured soon after the infusion of rtPA.

To the best of our knowledge, this is the first report to describe a rupture of an aneurysm in the non-infarcted hemisphere soon after an intravenous infusion of rtPA. There have been only three case reports describing aneurysmal SAH that developed after the infusion of rtPA. In the first reported case, ${ }^{8}$ the patient experienced a thunderclap headache three days before there was a presentation with ischemic stroke. Thus, the patient may have experienced a previous hemorrhage and the re-bleeding was precipitated by rtPA. The second case described a patient with a dissecting aneurysm, ${ }^{9}$ which did not suggest that an incidental aneurysm ruptured following the infusion of rtPA. In the third case, a previously concealed thrombosed saccular aneurysm was revealed after recanalization. ${ }^{10}$ These reports, however, did not convincingly demonstrate that rtPA was the culprit for the ruptured incidental aneurysms. The current case suggests that the infusion of rtPA resulted in the rupture of an intracranial aneurysm in a patient with an acute ischemic stroke.

Several pathophysiological mechanisms may explain the rupture of intracranial aneurysm, after the administration of rtPA. A previous study reported that rtPA can increase turbulence inside an unruptured aneurysm by rapidly dissolving a previous thrombus located at aneurysmal sac. Increased turbulence inside an unruptured aneurysm has been suggested as a possible cause of rupture. ${ }^{12}$ Hemorrhagic transformation in the infarcted brain tissue after a rtPA infusion may be related to dysregulated extracellular proteolysis within the neurovascular matrix. In addition, a rtPA infusion can also induce alterations of vascular permeability through the disruption of the integrity of the vascular basal lamina. ${ }^{13}$

In conclusion, the current study demonstrates that an incidental intracranial aneurysm may rupture after an intravenous infusion of rtPA in patients who experience acute ischemic stroke. Although recent studies have demonstrated no increase in the risk for rtPA-related intracranial 
hemorrhage in acute ischemic stroke patients harboring intracranial aneurysm, we suggest that rupture-prone aneurysms, in terms of size and shape, would be considered as a relative contraindication to rtPA. Future studies are needed to investigate whether rtPA is beneficial or harmful in patients who have both acute ischemic stroke and intracranial aneurysms.

\section{REFERENCES}

1. Edwards NJ, Kamel H, Josephson SA. The safety of intravenous thrombolysis for ischemic stroke in patients with preexisting cerebral aneurysms: a case series and review of the literature. Stroke 2012;43:412-6.

2. Vlak MH, Algra A, Brandenburg R, Rinkel G). Prevalence of unruptured intracranial aneurysms, with emphasis on sex, age, comorbidity, country, and time period: a systematic review and meta-analysis. Lancet Neurol 2011;10:626-36.

3. Oh YS, Lee SI, Shon YM, Yang DW, Kim BS, Cho AH. Incidental unruptured intracranial aneurysms in patients with acute ischemic stroke. Cerebrovasc Dis 2008;26:650-3.

4. Jauch EC, Saver JL, Adams HP Jr, Bruno A, Connors JJ, Demaerschalk BM, et al. Guidelines for the early management of patients with acute ischemic stroke: a guideline for healthcare professionals from the American Heart Association/ American Stroke Association. Stroke 2013;44:870-947.

5. Goyal N, Tsivgoulis G, Zand R, Sharma VK, Barlinn K, Male S, et al. Systemic thrombolysis in acute ischemic stroke pa- tients with unruptured intracranial aneurysms. Neurology 2015;85:1452-8.

6. Sheth KN, Shah N, Morovati T, Hermann LD, Cronin CA. Intravenous rt-PA is not associated with increased risk of hemorrhage in patients with intracranial aneurysms. Neurocritical Care 2012;17:199-203.

7. Mittal MK, Seet RC, Zhang Y, Brown RD Jr, Rabinstein AA. Safety of intravenous thrombolysis in acute ischemic stroke patients with saccular intracranial aneurysms. / Stroke Cerebrovasc Dis 2013;22:639-43.

8. Rammos SK, Neils DM, Fraser K, Klopfenstein JD. Anterior communicating artery aneurysm rupture after intravenous thrombolysis for acute middle cerebral artery thromboembolism: case report. Neurosurgery 2012;70:E1603-7; discussion E1607.

9. Haji F, van Adel BV, Avery M, Megyesi J, Young GB. Intracranial aneurysm rupture following intravenous thrombolysis for stroke. Can / Neurol Sci 2014;41:95-8.

10. Briosa E Gala D, Almeida A, Monteiro N, Paiva Nunes A, Ferreira $\mathrm{P}$, Mendonça $\mathrm{N}$, et al. Successful Thrombolysis despite Having an Incidental Unruptured Cerebral Aneurysm. Case Rep Neurol Med 2014;2014:323049.

11. Yoo SH, Kwon SU, Lee DH, Kim SJ, Kim IS, Kang DW. Comparison between MRI screening and CT-plus-MRI screening for thrombolysis within 3 h of ischemic stroke. / Neurol Sci 2010;294:119-23.

12. Lagares A, Gómez PA, Lobato RD, Alén JF, Campollo J, BenitoLeón J. Cerebral aneurysm rupture after r-tPA thrombolysis for acute myocardial infarction. Surg Neurol 1999;52:623-6.

13. Chalouhi N, Hoh BL, Hasan D. Review of cerebral aneurysm formation, growth, and rupture. Stroke 2013;44:3613-22. 